\title{
The genetics of the metabolic overlap syndrome
}

\author{
James Scott, C. Shoulders, N. Navaratnam \& T. Aitman \\ Imperial College School of Medicine, Hammersmith Hospital, Du-Cane Road, London W12 ONN, UK
}

The metabolic overlap syndromes hyperlipidaemia, diabetes mellitus, insulin resistance, obesity and hypertension together constitute the major risk factors for CHD. The genetic predisposition to these conditions is strong but complex, and many of the genes involved have not been identified. The identification of the genes or metabolic pathways involved in these disorders would lead to new diagnostic and therapeutic targets with wide applicability. The spontaneously-hypertensive rat (SHR) is a model of essential hypertension which also displays abnormalities of lipid metabolism and insulin resistance similar to those found in the human metabolic overlap syndromes. These abnormalities include raised blood triacylglycerols and fatty acids, defective catecholamine-mediated lipolysis and excessive growth of intra-abdominal adipocytes. Similar abnormalities have been identified in combined hyperlipidaemia, maturity-onset diabetes mellitus and obesity in human subjects. We are using the SHR as a model to identify genes and pathways which are likely to be in common with those causing the human diseases. We have recently identified loci on chromosomes 4 and 12 in SHR that confer insulin resistance. The chromosome 4 locus also confers abnormalities of catecholamine-mediated lipolysis. The chromosome 4 locus has been mapped using radiation hybrids, and the lipid and insulin resistance abnormalities isolated in a congenic rat strain. Using cDNA microarrays and radiation hybrid mapping, we have now identified a defective SHR gene that resides at the peak of linkage to these SHR quantitative trait loci. The SHR coding sequence for this gene contains multiple sequence variants caused by unequal recombination of a duplicated ancestral gene. The gene encodes a plasma-membrane protein on the peroxisome proliferator-activated receptor $\gamma$ pathway. The encoded protein product is undetectable in SHR adipocyte plasma membrane. The known biological functions of this protein suggest that this gene underlies some, if not all, these SHR quantitative trait loci phenotypes. CD36 is an important candidate for susceptibility to the human insulinresistance syndromes. The human gene is being examined for linkage and association in human subjects with insulin resistance from different ethnic backgrounds and subjects with combined hyperlipidaemia. The results of these studies, together with the results of recent linkage studies in familial combined hyperlipidaemia, will be presented.

Coronary heart disease: Metabolic overlap syndromes: Spontaneously-hypertensive rat 